\title{
Trend of caesarean deliveries in Egypt and its associated factors: evidence from national surveys, 2005-2014
}

Rami H. Al Rifai

\begin{abstract}
Background: The continued rise in caesarean section (c-section) deliveries raises a major public health concern worldwide. This study assessed the trend of c-section deliveries and examined factors associated with a rise in csection deliveries among the Egyptian mothers, from 2005 to 2014, by place of delivery.

Methods: This study utilized the 2005, 2008, and 2014 Egypt Demographic and Health Surveys (EDHS). The EDHS reported on the mode of delivery for the last birth occurred within five years preceding each survey including place of delivery and sociodemographic information for a total sample of over 29,000 mothers in the three surveys. To document trend of c-section, the EDHS-2005 was set as a reference in two binary logistic regression models; among all mothers together and for mothers stratified by place of delivery (public or private). $P$-value for the trend was assessed by entering the year of the survey as a continuous variable. The study followed STROBE statement in reporting observational studies.
\end{abstract}

Results: Institutional-based c-sections increased by 40.7 points from EDHS-2005 to EDHS-2014 (aOR, 3.46, 95\%Cl: 3.15-3. $\left.80, P_{\text {trend }}<0.001\right)$. Compared to mothers with low socioeconomic status (SES), mothers with high SES had higher odds (aOR, 1.78, 95\%Cl: 1.25-2.54, $P=0.001$ ) for c-section, but only in EDHS-2005. The adjusted trend of c-sections was found to be 4.19-time (95\%Cl: 3.73-4.70, $P<0.001$ ) higher in private sector while that in public sector it was 2.67 -time $(95 \% \mathrm{Cl}$ : $2.27-$ $3.13, P=0.001$ ) higher, in EDHS-2014 relative to EDHS-2005. This increase in the private sector is explained by significant increases among mothers who are potentially at low risk for c-sections; mothers aged 19-24 years vs. $\geq 35$ years (aOR: 0.31, 95\%Cl: $0.21-0.45$, in EDHS-2005 vs. 0.43 , 95\%Cl: $0.33-0.56$, in EDHS-2014, $P<0.001$ ); primigravida mothers vs. mothers with $\geq 4$ children (aOR: 1.62, 95\%Cl: 1.12-2.34, in EDHS-2005 vs. 3.76, 95\%Cl: 2.94-4.80 in EDHS-2014); and among normal compared to high risk birth weight babies (aOR: 0.79, 95\%Cl: 0.62-0.99 in EDHS-2005 $P<0.05$ vs. 0.83, 95\%Cl: $0.65-1.04$ in EDHS-2014, P> 0.05).

Conclusions: Results showed a steady rise in c-sections in Egypt that has reached an alarming level in recent years. This increase appears to be associated with a shift towards delivery in private health care facilities. More vigilance of c-section deliveries, particularly in the private sector, is warranted.

Keywords: Egypt, Caesarean section delivery, Maternal health, Maternal delivery

Correspondence: rrifai@uaeu.ac.ae

Institute of Public Health, College of Medicine and Health Sciences, United

Arab Emirates University, Al-Ain, United Arab Emirates 


\section{Background}

Caesarean section (c-section) delivery is a major surgical operation aimed at saving lives [1]. Globally, the proportion of c-sections, one of the most common surgeries, continues to rise particularly in high- and middle-income countries [1]. Caesarean sections should be performed when vaginal delivery poses a risk to the mother or baby as in case of prolonged labor, fetal distress, or fetal malpresentation. The World Health Organization (WHO) underscores the importance of focusing on the needs of the pregnant mothers and discourages performing c-sections with no need. Caesarean delivery without a medical need places mothers and their babies at-risk of short- and longterm health consequences [1]. It is associated with increased risk of blood transfusion, hysterectomy, maternal and child death [2-4], uterine rupture, placenta accreta, and placenta previa $[5,6]$. It also costs more and requires longer hospitalization than vaginal delivery [7].

The WHO has considered a population-based rate of c-sections between 10 and 15\%, as an ideal rate that was associated with a notable decline in maternal mortality ratio (MMR) and neonatal mortality rate (NMR) [1]. In 2008, half of 137 countries have exceeded this recommended threshold [8]. In 23 out of 24 countries, proportion of c-sections without medical indication ranged between $0.01 \%$ and $2 \%$ [3]. Caesarean section rate varied across different countries, worldwide. The rate was between $20 \%$ and $22.5 \%$ in the United Kingdom and Canada. In Italy and South America, c- section rate was as high as $85 \%$ [9-11]. In Saudi Arabia, the c-section rate increased by $80 \%$, from $10.6 \%$ to $19.1 \%$, between the years 1997 and 2006 [12]. Private practice has contributed to the increased c- section rate internationally. In Rome, the rate of c-section delivery was reported to be approximately 44\%; $85 \%$ of all birth in some private clinics of Rome were c-section [13]. In Jordan, a neighboring country to Egypt, between 2002 and 2012, the csection deliveries increased significantly over time, from $18.2 \%$ in 2002 to $30.3 \%$ in 2012 ; an increase by $70 \%$ in csections was in the private hospitals [14].

Health services in Egypt are provided by three sectors based on the financing source: the public, the parastatal, and the private sector. The public sector covers the government and quasi-governmental hospitals (parastatal). Public hospitals receive funding from the Ministry of Finance while the government ministries have a controlling share of decision making in parastatal hospitals [15]. Public hospitals include a total of 1,048 inpatient facilities with more than 80,000 beds. The private sector has a total of 2,024 inpatient facilities with a total of 22,647 beds that accounts for approximately $16 \%$ of the total inpatient bed capacity in Egypt [15].

In the past two decades, there were significant achievements in matters related to maternal health in
Egypt. Home-based deliveries declined by over 60\% [16], medically assisted births rose sharply from 35\% in 1988 to $92 \%$ in 2014 , and $90 \%$ of mothers received antenatal care (ANC) services from a trained provider [16]. The MMR declined from 174/100,000 live births in 1992 to $54 / 100,000$ in 2010 [17]. However, over the last two years, the MMR has slightly increased to $57 / 100,000$; $23.5 \%$ of these mortalities delivery were initiated in a private clinic [17]. The NMR was 14/1,000 births during the five-year period prior to 2014 [16].

The last study tracking changes in c-sections in Egypt was reported in 2004 [18]. According to which, based on data from two Egyptian Demographic and Health Surveys (EDHS), the institutional-based proportion of c-sections increased from $13.9 \%$ in 1988 to $22 \%$ in 2000 [18]. Birth delivery in the private sector was associated with this increase [18]. However, this study did not explore the change in c-section rate among mothers who are potentially at low risk for c-section. Although EDHS is a noninstitutional-based survey, it is reported that the DHS data on c-section deliveries are sufficiently reliable for national and global monitoring purposes since the recall bias on reporting a major surgical procedure is very low [19]. With the increased access to healthcare services in Egypt, represented by a decline in home-based deliveries and an increase in ANC services [16], the specific objectives of this study are: to (1) assess the trend of c-section deliveries and (2) to identify factors associated with the over time change in c-sections in Egypt, from 2005 to 2014, with a focus on the role of place of delivery in performing csections, particularly for mothers who are potentially at low risk for c-section delivery.

\section{Methods}

\section{Data sources}

The EDHS survey aimed at providing national estimates with special emphasis on maternal and child health [16]. Employing a standardized and rigorous sampling and data collection methodology, the survey collected information from a nationally representative sample of Egyptian individuals with a large sample size [16]. Details related to sampling design, sample size, study instruments, data collection, how informed consent was obtained, and other related methodology are described elsewhere [16]. The EDHS data are accessible from the Measure DHS website [20]. The STROBE statement for reporting observational studies was followed [21] (STROBE checklist can be found in the Additional file 1).

\section{Study population}

For the purpose of this study, the 2005, 2008, and 2014 EDHS ever-married women databases were merged based on established guidelines for managing DHS data [22]. All mothers who replied by "yes" or "no" to the 
question "Has your last baby born in the past five years, including this year, was delivered by a c-section or normal/vaginal delivery?" were included in the analysis, leaving a final sample of 29,489 mothers (weighted sample of 29,107) after excluding five women with missing data and 28,269 women who did not report giving birth within the prior five years (Fig. 1).

\section{Study variables}

\section{Sociodemographics}

Specific information collected in the EDHS that reported $[14,18,23]$ or could potentially have an influence on the mode of birth delivery was characterized. Sociodemographic characteristics included mother's age at last birth $(\leq 18,19-24,25-29,30-34$, and $\geq 35$ years). Mother's socioeconomic status (SES) was defined based on composite scores of the mother's education level and household's wealth status. This was performed to address the problem of multicollinearity between education and income [24]. First, mothers were dichotomized according to their education level as either with "low education" (primary or below) or with "high education" (secondary or above) and trichotomized them according to their wealth status into tertiles: poor, middle, or rich. Details related to the EDHS wealth index can be found elsewhere [16]. Then, based on the sum of scores of education and wealth variables, mothers with low SES (poor and with low education), middle SES (either poor or with low education), and high SES (non-poor and with high education) were identified [24].

Mothers were categorized according to their lifetime parity into primigravida, multipara with $2-3$, or multipara with $\geq 4$ births. Female genital mutilation (FGM) status was reported in a binary variable (yes or no). Body mass index (BMI) $\left(\mathrm{kg} / \mathrm{m}^{2}\right)$ was calculated as the ratio of the weight in kilograms divided by height squared $\left(\mathrm{m}^{2}\right)$ data that were collected at the time of survey. Mother's BMI was trichotomized into normal $(\mathrm{BMI}=18.5$ $24.9 \mathrm{~kg} / \mathrm{m} 2)$, underweight (BMI $<18.5 \mathrm{~kg} / \mathrm{m} 2$ ), or overweight/obese (BMI $\geq 25 \mathrm{~kg} / \mathrm{m} 2$ ) [25]. Maternal BMI has been linked to increased risk of adverse pregnancy outcomes leading to c-section delivery. Underweight and overweight/obese mothers were defined as a "high-risk" group for c-section. Underweight mothers are more likely to have underweight babies and pre-term delivery [26] while overweight/obese mothers are more likely to have overweight babies, preeclampsia, macrosomia, and gestational diabetes [27-30].

\section{Spatial characteristics}

Spatial characteristics included place of residence (rural or urban) and geographical region (urban governorates, urban or rural-lower Egypt, and urban or rural-upper Egypt).

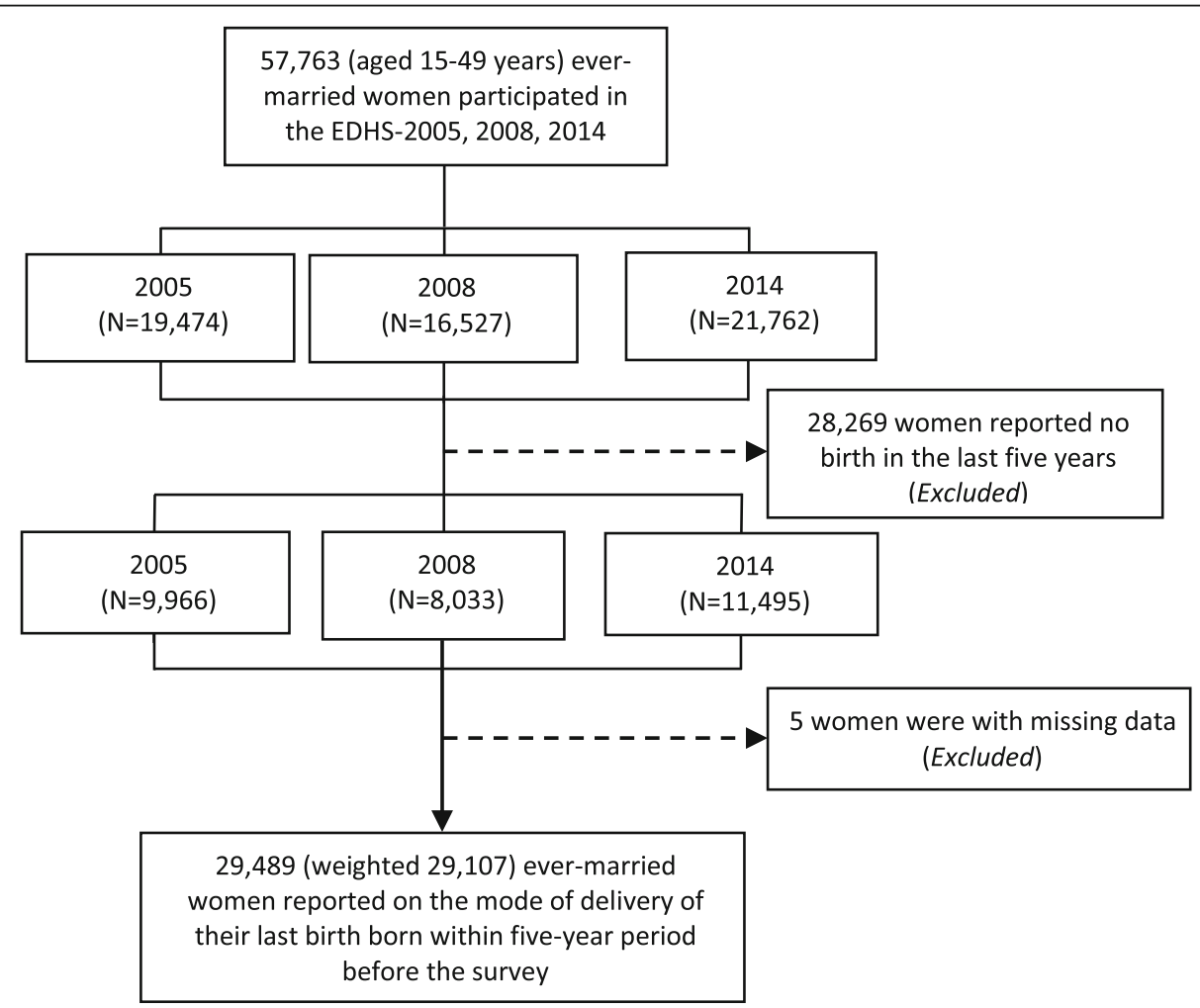

Fig. 1 Sample selection, unweighted numbers 


\section{Newborn characteristics}

Mothers were categorized according to the birth weight as mother with: normal (2,500-3,999 g), low $(<2,500 \mathrm{~g})$, or high $(\geq 4,000 \mathrm{~g})$ birth weight baby. According to the birth multiplicity of the last birth born within the 5 years prior to the EDHS survey, mothers were categorized into two groups (singleton or multiple births). Mothers reported singleton or normal birth weight (2,500 to $3,999 \mathrm{~g}$ ) babies were categorized as "low risk" while multiple or low/high birth weight $(<2,500 \mathrm{~g}$ or $\geq 4,000 \mathrm{~g})$ babies were categorized as a "high risk" group for c-section $[14,23]$. Low/high birth weight or multiple births are main obstetric variables to increase risk of c-section [23, 31]. The mode of last birth delivery in relation to mode of delivery of the previous birth occurred within the five years of survey was categorized into vaginal after csection, repeated c-section, repeated vaginal, or c-section after vaginal. Primigravida mothers were also included within this variable.

\section{Institutional characteristics}

According to the number of ANC visits made by mother during her last pregnancy, mothers were categorized into four groups (no visits, 1-3, $\geq 4$, or don't know/missing). Place of birth delivery was categorized into: home, public sector including parastatal health care facilities, or private sector.

\section{Statistical analyses}

Frequency of mothers for each survey and their percentage distribution per each measured characteristic for all births and for institutional-based births were calculated, by survey round. Also, the proportion of institutionalbased c-sections for each measured characteristic by survey round were recorded and assessed the over time trend of c-sections for each measured category's item by chi-square tests for trend.

The crude and adjusted trend of c-sections utilizing the EDHS-2005 as a reference in binary and multivariable logistic regression models for all institutional-based deliveries, and stratified by place of delivery, after merging the datasets were subsequently investigated. This stratification was done to examine the role of place of delivery on the over time trend of c-sections in Egypt. In the binary logistic regression models, $P$-value for the trend was assessed for each model by entering the year of the survey as a continuous variable.

The quantitative association between institutionalbased c-sections and each measured characteristic for each survey separately was also investigated. The crude (ORs) and adjusted odds ratios (aORs) and their respective $95 \%$ confidence intervals (CIs) were reported. To adjust for any potential confounding effect, all covariates were entered under analysis simultaneously in multivariable models.

A place of delivery-stratified multivariable logistic regression analysis was performed to quantify the association between institutional-based c-sections and the measured characteristics for each survey round. This was performed to understand which factors were associated with increased trend of c-sections in Egypt, stratified by place of delivery.

The sampling weights available in the EDHS databases were applied in all of performed statistical calculations. The data were analyzed using IBM SPSS version 22.0 (IBM Corp., Armonk, NY, USA) [32]. P-values <0.05 were considered statistically significant.

\section{Results}

The three EDHSs were based on a nationally representative sample of 57,763 ever-married women aged 15 to 49 years, of which, 29,107 mothers reported on the mode of delivery of their last birth born within five years preceding each survey (Fig. 1).

Table 1 presents descriptive characteristics of all mothers and mothers who reported institutional-based delivery by survey round. The table shows that the institutional-based last birth occurred at mother's age of $\geq 35$ years or $\leq 18$ years declined slightly from $11.4 \%$ to $10.2 \%$ and from $4.9 \%$ to $3.7 \%$, from EDHS-2005 to EDHS-2014, respectively. Percentage of mothers with low SES who reported institutional-based birth declined by 4.9 percentage points mainly for an increase by 3.9 percentage points for the favor of mothers with a medium SES. Percentage of primigravida or mothers with $\geq 4$ children declined from $28.2 \%$ to $24.3 \%$ and from $21.7 \%$ to $20.7 \%$, respectively. Percentage of overweight mothers increased from $76.2 \%$ to $79.6 \%$. Over the study period, percentage of institutional-based normal birth weight babies increased by 13.6 percentage points. Institutional-based c-sections increased from $32.4 \%$ in EDHS-2005 to $61.8 \%$ in EDHS-2014. Repeated c-sections increased by 10 percentage points along a slight increase in $\mathrm{c}$-sections after vaginal delivery by only 1.1 percentage points. Birth delivery declined in public sector by 11.8 percentage points for the favor of the private sector.

Proportion of c-sections by year showed that the population-based trend of c-sections increased exponentially from $17.8 \%$ in 2000 to $59.7 \%$ in 2014 that further increased from $26.6 \%$ to $67.3 \%$ when the analysis was limited to the institutional-based deliveries, during the same study period (Fig. 2).

Table 2 shows the over time changes in proportion of institutional-based c-sections. From EDHS-2005 to EDHS-2014, proportion of c-sections increased among mothers in each measured characteristic within each measured category. Largest increases were among 
Table 1 Descriptive characteristics of all mothers $(N=29,107)$ and mothers with the last institutional-based $(N=22,194)$ birth occurred within five years prior to each survey, by survey round

\begin{tabular}{|c|c|c|c|c|c|}
\hline \multicolumn{2}{|c|}{ EDHS-2005 } & \multicolumn{2}{|l|}{ EDHS-2008 } & \multicolumn{2}{|l|}{ EDHS-2014 } \\
\hline $\begin{array}{l}\text { All births } \\
N=9823\end{array}$ & $\begin{array}{l}\text { Institutional } \\
\text { births } N=6517\end{array}$ & All births $N=7893$ & $\begin{array}{l}\text { Institutional } \\
\text { births } N=5713\end{array}$ & $\begin{array}{l}\text { All births } \\
N=11,391\end{array}$ & $\begin{array}{l}\text { Institutional } \\
\text { births } N=9964\end{array}$ \\
\hline$\%$ & $\%$ & $\%$ & $\%$ & $\%$ & $\%$ \\
\hline
\end{tabular}

Socio-demographic

Age at last birth, years

$\begin{array}{llllll}\geq 35 & 11.7 & 11.4 & 11.1 & 11.2 & 10.2 \\ 30-34 & 18.2 & 18.3 & 16.6 & 16.4 & 18.5 \\ 25-29 & 29.2 & 30.3 & 30.6 & 31.2 & 32.9 \\ 19-24 & 36.0 & 35.1 & 36.8 & 36.3 & 33.8 \\ \leq 18 & 4.9 & 4.9 & 4.9 & 4.8 & 3.7\end{array}$

Socioeconomic status

Low

Medium

High

Lifetime parity

Multipara with $\geq 4$ children

Multipara with 2-3 children

Primigravida

FGM $^{a}$

No

Yes

Missing

BMI

Normal 18.5-24.9 (low risk)

25.7

Underweight $<18.5$ (high risk)

Overweight $\geq 25$ (high risk)

Missing

Spatial

Residence

Urban

Rural

Region

Urban governorates

Urban-Lower Egypt

Rural-Lower Egypt

Urban-Upper Egypt

Rural-Upper Egypt

Frontier governorates

Newborn characteristics

Birth weight

Normal (2,500-3,999 g)

High risk $(<2,500, \geq 4,000 \mathrm{~g})$

Not weighted/Missing
28.4

18.7

24.5

56.8

21.7

50.1

28.2

4.9

95.1
22.3

0.5

76.2

1.1

48.0

38.1

19.7

13.1

32.2

14.4

19.4

1.1

8.7

10.8

50.2

28.7

0.6

70.1

0.6

60.8
35.1

61.9

52.0

\author{
.
}

0

8.1

56.8
4.7

16.4
10.1
34.3
10.8
27.3
1.4

23.7

49.7

26.6

95.3

5.7

94.3

16.6

23.5

60.0

19.2

50.2

30.6

0.6

5.7

4.3

25.5

0.4

73.4

0.7

45.3

54.7

20.4

12.0

35.4

11.9

18.8

1.5

43.5

49.4

52.6

10.1

13.5

14.1

46.3
13.8

28.4

57.9

20.7

55.0

24.3

9.2

90.7

19.3

0.3

79.6

0.8

$\begin{array}{ll}0.8 & 0.8\end{array}$


Table 1 Descriptive characteristics of all mothers $(N=29,107)$ and mothers with the last institutional-based $(N=22,194)$ birth occurred within five years prior to each survey, by survey round (Continued)

\begin{tabular}{|c|c|c|c|c|c|c|}
\hline & \multicolumn{2}{|c|}{ EDHS-2005 } & \multicolumn{2}{|l|}{ EDHS-2008 } & \multicolumn{2}{|l|}{ EDHS-2014 } \\
\hline & $\begin{array}{l}\text { All births } \\
N=9823\end{array}$ & $\begin{array}{l}\text { Institutional } \\
\text { births } N=6517\end{array}$ & All births $N=7893$ & $\begin{array}{l}\text { Institutional } \\
\text { births } N=5713\end{array}$ & $\begin{array}{l}\text { All births } \\
N=11,391\end{array}$ & $\begin{array}{l}\text { Institutional } \\
\text { births } N=9964\end{array}$ \\
\hline & $\%$ & $\%$ & $\%$ & $\%$ & $\%$ & $\%$ \\
\hline \multicolumn{7}{|l|}{ Birth multiplicity } \\
\hline Singleton birth & 97.6 & 97.0 & 97.9 & 97.4 & 97.8 & 97.6 \\
\hline Multiple birth & 2.4 & 3.0 & 2.1 & 2.6 & 2.2 & 2.4 \\
\hline \multicolumn{7}{|l|}{ Delivery mode } \\
\hline Vaginal & 78.5 & 67.6 & 70.8 & 59.7 & 45.9 & 38.2 \\
\hline C-section & 21.5 & 32.4 & 29.2 & 40.3 & 54.1 & 61.8 \\
\hline \multicolumn{7}{|c|}{ Delivery mode in relation to the previous ${ }^{b}$} \\
\hline Primigravida & 66.0 & 69.0 & 69.0 & 70.5 & 66.2 & 66.8 \\
\hline Vaginal after caesarean & 0.5 & 0.4 & 0.4 & 0.4 & 0.4 & 0.3 \\
\hline Repeated caesarean & 5.2 & 7.8 & 6.9 & 9.5 & 15.6 & 17.8 \\
\hline Repeated vaginal & 27.0 & 20.9 & 22.2 & 17.5 & 15.2 & 12.1 \\
\hline Caesarean after vaginal & 1.3 & 1.9 & 1.5 & 2.1 & 2.6 & 3.0 \\
\hline \multicolumn{7}{|l|}{ Institutional } \\
\hline \multicolumn{7}{|l|}{ Antenatal care visits } \\
\hline No visits & 28.5 & 18.3 & 25.8 & 17.1 & 9.7 & 7.3 \\
\hline $1-3$ & 10.1 & 8.3 & 6.9 & 6.0 & 7.1 & 6.1 \\
\hline$\geq 4$ & 60.7 & 72.8 & 66.5 & 76.2 & 82.8 & 86.2 \\
\hline Don't know/missing & 0.7 & 0.6 & 0.8 & 0.7 & 0.4 & 0.4 \\
\hline \multicolumn{7}{|l|}{ Place of delivery } \\
\hline Home & 33.6 & 0.0 & 27.6 & 0.0 & 12.5 & 0.0 \\
\hline Public sector & 26.9 & 40.6 & 27.2 & 37.6 & 25.2 & 28.8 \\
\hline Private sector & 39.5 & 59.4 & 45.2 & 62.4 & 62.3 & 71.2 \\
\hline Missing & & 4 & & 3 & & \\
\hline
\end{tabular}

Weighted numbers and percentages

BMl: body mass index

${ }^{a}$ female genital mutilation, ${ }^{b}$ previous birth occurred within past five years

mothers aged $\leq 18$ years (34.1 points), primigravida mothers (34.4 points), mothers with normal BMI (31.4 points), normal birth weight (30.1 points) or singleton babies (29.5 points), and in the private sector (32.6 points). Of the 6158 institutional-based c-sections performed in EDHS-2014, 77.3\% were performed in the private sector.

The multivariable analysis revealed a 3.46-time $(95 \%$ CI: 3.15-3.80) increase in trend of c-sections in EDHS2014 relative to EDHS-2005. The further place of delivery-stratified multivariate analysis showed that this increase in trend of c-sections was higher in private sector (aOR, 4.19, 95\% CI: 3.73-4.70) than in public sector (aOR, 2.67, 95\% CI: 2.27-3.13) (Table 3).

Table 4 shows the results of bivariate and multivariable logistic regression of association between institutionalbased c-section and measured characteristics by survey round. As the table shows, relative to mothers aged $\geq 35$ years, in EDHS-2014, the trend of c-section among

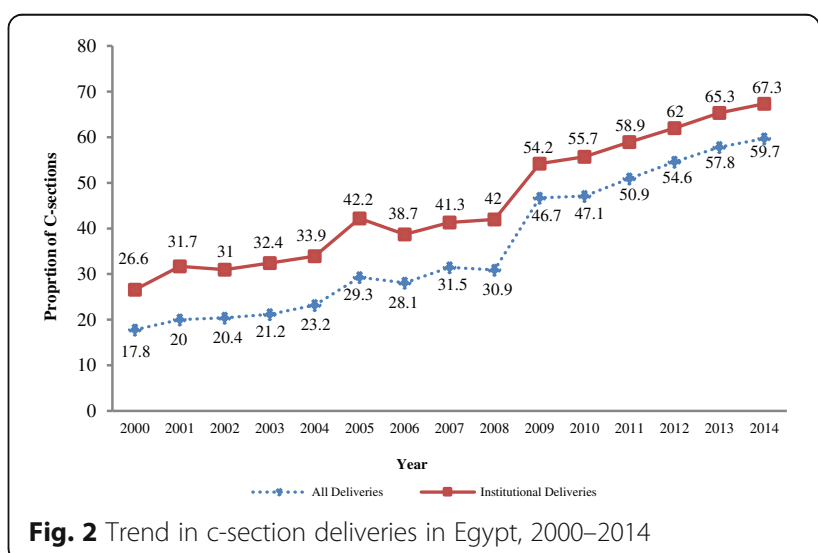


Table 2 Institutional-based proportion of c-section deliveries among mothers with a last birth occurred within five years prior to each survey, by survey round

\begin{tabular}{|c|c|c|c|c|c|c|}
\hline & EDHS-2005 N = 6517 & EDHS-2008 N = 5713 & $P$-value ${ }^{a}$ & EDHS-2014 N = 9964 & $P$-value ${ }^{b}$ & Absolute difference \\
\hline & C-section, \% & C-section, \% & & C-section, \% & & (\% in 2014-\% in 2005 \\
\hline \multicolumn{7}{|l|}{ Socio-demographic } \\
\hline \multicolumn{7}{|l|}{ Age at last birth } \\
\hline$\geq 35$ & 37.2 & 44.3 & 0.007 & 62.4 & $<0.001$ & 25.2 \\
\hline $30-34$ & 35.5 & 43.1 & $<0.001$ & 62.4 & $<0.001$ & 26.9 \\
\hline $25-29$ & 33.6 & 42.3 & $<0.001$ & 61.5 & $<0.001$ & 27.9 \\
\hline $19-24$ & 29.2 & 37.1 & $<0.001$ & 61.8 & $<0.001$ & 32.6 \\
\hline$\leq 18$ & 25.8 & 33.6 & 0.038 & 59.9 & $<0.001$ & 34.1 \\
\hline$P$-value ${ }^{b}$ & $<0.001$ & $<0.001$ & & 0.903 & & \\
\hline \multicolumn{7}{|l|}{ Socioeconomic status } \\
\hline Low & 22.7 & 33.5 & $<0.001$ & 51.6 & $<0.001$ & 28.9 \\
\hline Medium & 29.2 & 35.2 & 0.001 & 57.9 & $<0.001$ & 28.7 \\
\hline High & 37.0 & 44.2 & $<0.001$ & 66.1 & $<0.001$ & 29.1 \\
\hline$P$-value ${ }^{b}$ & $<0.001$ & $<0.001$ & & $<0.001$ & & \\
\hline \multicolumn{7}{|l|}{ Lifetime parity } \\
\hline Multipara with $\geq 4$ children & 27.3 & 33.3 & 0.001 & 52.2 & $<0.001$ & 24.9 \\
\hline Multipara with 2-3 children & 32.9 & 40.8 & $<0.001$ & 61.8 & $<0.001$ & 28.9 \\
\hline Primigravidas & 35.6 & 43.9 & $<0.001$ & 70.0 & $<0.001$ & 34.4 \\
\hline$P$-value ${ }^{b}$ & $<0.001$ & $<0.001$ & & $<0.001$ & & \\
\hline \multicolumn{7}{|l|}{$\mathrm{FGM}^{c}$} \\
\hline No & 44.8 & 53.5 & 0.027 & 68.9 & $<0.001$ & 24.1 \\
\hline Yes & 31.8 & 39.5 & $<0.001$ & 61.1 & $<0.001$ & 29.3 \\
\hline$P$-value ${ }^{b}$ & $<0.001$ & $<0.001$ & & $<0.001$ & & \\
\hline Missing & 5 & 0 & & 3 & & \\
\hline \multicolumn{7}{|l|}{ BMI } \\
\hline Normal (18.5-24.9) & 26.2 & 36.3 & $<0.001$ & 57.6 & $<0.001$ & 31.4 \\
\hline High risk $(<18.5, \geq 25)$ & 34.2 & 41.6 & $<0.001$ & 62.8 & $<0.001$ & 28.6 \\
\hline$P$-value ${ }^{b}$ & $<0.001$ & $<0.001$ & & $<0.001$ & & \\
\hline Missing & 69 & 41 & & 80 & & \\
\hline \multicolumn{7}{|l|}{ Spatial } \\
\hline \multicolumn{7}{|l|}{ Residence } \\
\hline Urban & 36.8 & 44.5 & $<0.001$ & 65.8 & $<0.001$ & 29.0 \\
\hline Rural & 28.4 & 36.9 & $<0.001$ & 59.7 & $<0.001$ & 31.3 \\
\hline$P$-value ${ }^{b}$ & $<0.001$ & $<0.001$ & & $<0.001$ & & \\
\hline \multicolumn{7}{|l|}{ Region } \\
\hline Urban governorates & 40.4 & 44.6 & 0.034 & 67.3 & $<0.001$ & 26.9 \\
\hline Urban-Lower Egypt & 42.0 & 48.8 & 0.008 & 73.6 & $<0.001$ & 31.6 \\
\hline Rural-Lower Egypt & 32.3 & 38.4 & $<0.001$ & 65.8 & $<0.001$ & 33.5 \\
\hline Urban-Upper Egypt & 27.6 & 41.2 & $<0.001$ & 57.8 & $<0.001$ & 30.2 \\
\hline Rural-Upper Egypt & 22.2 & 34.4 & $<0.001$ & 50.2 & $<0.001$ & 28.0 \\
\hline Frontier governorates & 24.7 & 28.9 & 0.550 & 51.6 & 0.002 & 26.9 \\
\hline$P$-value ${ }^{b}$ & $<0.001$ & $<0.001$ & & $<0.001$ & & \\
\hline \multicolumn{7}{|l|}{ Newborn characteristics } \\
\hline \multicolumn{7}{|l|}{ Birth weight } \\
\hline Normal $(2,500-3,999)$ & 35.1 & 41.4 & $<0.001$ & 65.2 & $<0.001$ & 30.1 \\
\hline High risk $(<2,500, \geq 4,000)$ & 41.6 & 51.1 & 0.001 & 67.1 & $<0.001$ & 25.5 \\
\hline
\end{tabular}


Table 2 Institutional-based proportion of c-section deliveries among mothers with a last birth occurred within five years prior to each survey, by survey round (Continued)

\begin{tabular}{|c|c|c|c|c|c|c|}
\hline & \multirow{2}{*}{$\begin{array}{l}\text { EDHS-2005 N=6517 } \\
\text { C-section, \% }\end{array}$} & \multirow{2}{*}{$\begin{array}{l}\text { EDHS-2008 N = } 5713 \\
\text { C-section, \% }\end{array}$} & \multirow[t]{2}{*}{$P$-value ${ }^{a}$} & \multirow{2}{*}{$\begin{array}{l}\text { EDHS-2014 N =9964 } \\
\text { C-section, \% }\end{array}$} & \multirow[t]{2}{*}{$P$-value ${ }^{b}$} & \multirow{2}{*}{$\begin{array}{l}\text { Absolute difference } \\
\text { (\% in 2014-\% in } 2005\end{array}$} \\
\hline & & & & & & \\
\hline$P$-value ${ }^{b}$ & 0.002 & $<0.001$ & & 0.182 & & \\
\hline Not weighted/Missing & 3272 & 2648 & & 3317 & & \\
\hline \multicolumn{7}{|l|}{ Birth multiplicity } \\
\hline Singleton birth & 32.0 & 40.1 & $<0.001$ & 61.5 & $<0.001$ & 29.5 \\
\hline Multiple birth & 48.0 & 50.0 & 0.709 & 75.2 & $<0.001$ & 27.2 \\
\hline$P$-value ${ }^{b}$ & $<0.001$ & 0.016 & & $<0.001$ & & \\
\hline \multicolumn{7}{|c|}{ Mode of delivery in relation to the previous birth ${ }^{d}$} \\
\hline Primigravidas mother & 32.9 & 40.8 & $<0.001$ & 61.4 & $<0.001$ & 28.5 \\
\hline Vaginal after caesarean & - & - & - & - & - & \\
\hline Repeated caesarean & - & - & - & - & - & \\
\hline Repeated vaginal & - & - & - & - & - & \\
\hline C-section after vaginal & - & - & - & - & - & \\
\hline \multicolumn{7}{|l|}{ Institutional } \\
\hline \multicolumn{7}{|l|}{ Antenatal care visits } \\
\hline No visits & 18.8 & 30.1 & $<0.001$ & 42.4 & $<0.001$ & 23.6 \\
\hline $1-3$ & 26.7 & 34.1 & 0.019 & 48.6 & $<0.001$ & 21.9 \\
\hline$\geq 4$ & 36.4 & 43.3 & $<0.001$ & 64.4 & $<0.001$ & 28.0 \\
\hline$P$-value & $<0.001$ & $<0.001$ & & $<0.001$ & & \\
\hline Don't know/missing & 40 & 41 & & 36 & & \\
\hline \multicolumn{7}{|l|}{ Place of delivery } \\
\hline Public sector & 29.4 & 35.4 & $<0.001$ & 48.5 & $<0.001$ & 19.1 \\
\hline Private sector & 34.4 & 43.2 & $<0.001$ & 67.0 & $<0.001$ & 32.6 \\
\hline$P$-value ${ }^{b}$ & $<0.001$ & $<0.001$ & & $<0.001$ & & \\
\hline
\end{tabular}

a $P$-value, assessed over time differences in proportion of institutional-based caesarean delivery between EDHS-2005 and EDHS-2008, and between EDHS-2008 and EDHS-2014

${ }^{\mathrm{b}} P$-value, assessed differences in proportion of institutional-based caesarean delivery between each measured sub-categories in each survey

c female genital mutilation

${ }^{d}$ previous birth occurred within the past five years

mothers aged $\leq 18$ years (aOR, 0.33, 95\% CI: $0.23-0.47$ ) or 19-25 years (aOR, 0.41, 95\% CI: 0.31-0.55) was higher when it compared with c-sections in EDHS-2005 (aOR, 0.26, 95\% CI: $0.16-0.50$ and 0.31 , 95\% CI: $0.22-$ 0.42, respectively). In EDHS-2005, mothers from high
SES (aOR, $1,78,95 \%$ CI: $1.25-2.54, P=0.001$ ) were more likely to undergo c-section relative to those with low SES, but in EDHS-2014 this observed significant difference disappeared. Among primigravida, trend of csections in EDHS-2014 (aOR, 5.57, 95\% CI: 4.46-6.97)

Table 3 Bivariate and multivariate logistic regression for the trend of institutional-based c-section delivery among all mothers, stratified by place of delivery

\begin{tabular}{|c|c|c|c|c|}
\hline Among all mothers & OR $(95 \% \mathrm{Cl})$ & & aOR (95\% Cl) & \\
\hline \multicolumn{5}{|l|}{ Survey round (Ref: EDHS-2005) } \\
\hline EDHS-2008 & $1.41(1.31-1.52)^{* * *}$ & & $1.39(1.25-1.54)^{* * *}$ & \\
\hline EDHS-2014 & $3.37(3.20-3.60)^{* * *}$ & & $3.46(3.15-3.80)^{* * *}$ & \\
\hline Place of delivery & Public sector & & Private sector & \\
\hline Survey round (Ref: EDHS-2005) & OR $(95 \% \mathrm{Cl})$ & aOR $(95 \% \mathrm{Cl})$ & OR $(95 \% \mathrm{Cl})$ & $\mathrm{aOR}(95 \% \mathrm{Cl})$ \\
\hline EDHS-2008 & $1.32(1.16-1.49)^{* * *}$ & $1.35(1.13-1.61)^{* *}$ & $1.45(1.33-1.60)^{* * *}$ & $1.42(1.25-1.62)^{* * *}$ \\
\hline EDHS-2014 & $2.26(2.02-2.53)^{* * *}$ & $2.67(2.27-3.13)^{* *}$ & $2.88(3.58-4.21)^{* * *}$ & $4.19(3.73-4.70)^{* * *}$ \\
\hline
\end{tabular}

OR: odds ratio, aOR: adjusted odds ratio (for all covariates under analysis), Cl: confidence interval

$P$-value for the trend was assessed by entering the year of the survey as a continuous variable

${ }^{*} P<0.05,{ }^{* *} P=0.001,{ }^{* * *} P<0.001$ 
Table 4 Bivariate and multivariate logistic regression for the association between institutional-based c-section as dependent variable and measured characteristics by the survey round

\begin{tabular}{|c|c|c|c|c|c|c|}
\hline \multirow[t]{2}{*}{ Characteristics } & \multicolumn{2}{|l|}{ EDHS-2005 } & \multicolumn{2}{|l|}{$\underline{E D H S}-2008$} & \multicolumn{2}{|l|}{ EDHS-2014 } \\
\hline & $\mathrm{OR}(95 \% \mathrm{Cl})$ & $\mathrm{aOR}(95 \% \mathrm{Cl})$ & OR $(95 \% \mathrm{Cl})$ & $\mathrm{aOR}(95 \% \mathrm{Cl})$ & $\mathrm{OR}(95 \% \mathrm{Cl})$ & $\mathrm{aOR}(95 \% \mathrm{Cl})$ \\
\hline \multicolumn{7}{|l|}{ Socio-demographic } \\
\hline \multicolumn{7}{|l|}{ Age at last birth (Ref: $\geq 35 \mathrm{yrs}$ ) } \\
\hline $30-34$ & $0.93(0.77-1.12)$ & $0.77(0.57-1.04)$ & $0.93(0.77-1.12)$ & $0.83(0.61-1.11)$ & $1.00(0.86-1.17)$ & $0.77(0.61-0.95)^{*}$ \\
\hline $25-29$ & $0.85(0.71-1.01)$ & $0.47(0.35-0.65)^{* * *}$ & $0.85(0.71-1.01)$ & $0.72(0.55-0.95)^{* *}$ & $0.97(0.84-1.11)$ & $0.54(0.44-0.66)^{* * *}$ \\
\hline $19-24$ & $0.70(0.59-0.83)^{* * *}$ & $0.31(0.22-0.42)^{* * *}$ & $0.70(0.59-0.83)^{* * *}$ & $0.41(0.31-0.55)^{* * *}$ & $0.98(0.85-1.13)$ & $0.41(0.31-0.55)^{* * *}$ \\
\hline$\leq 18$ & $0.58(0.44-0.78)^{* * *}$ & $0.26(0.16-0.50)^{* * *}$ & $0.63(0.47-0.85)^{* * *}$ & $0.37(0.23-0.60)^{* * *}$ & $0.90(0.71-1.15)$ & $0.33(0.23-0.47)^{* * *}$ \\
\hline \multicolumn{7}{|l|}{ Socioeconomic status (Ref: low) } \\
\hline Medium & $1.40(1.18-1.67)^{* * *}$ & $1.55(1.07-2.24)^{*}$ & $1.08(0.91-1.29)$ & $0.79(0.58-1.07)$ & $1.29(1.13-1.47)^{* * *}$ & $0.98(0.80-1.21)$ \\
\hline High & $2.0(1.72-2.32)^{* * *}$ & $1.78(1.25-2.54)^{* *}$ & $1.57(1.35-1.83)^{* * *}$ & $0.77(0.57-1.04)$ & $1.83(1.63-2.07)^{* * *}$ & $0.97(0.85-1.12)$ \\
\hline \multicolumn{7}{|c|}{ Lifetime parity (Ref: multipara with $\geq 4$ children) } \\
\hline Multipara with 2-3 children & $1.31(1.14-1.50)^{* * *}$ & $1.06(0.81-1.38)$ & $1.38(1.19-1.59)^{* * *}$ & $1.41(1.08-1.84)^{*}$ & $2.13(1.89-2.41)^{* * *}$ & $1.52(1.27-1.81)^{* * *}$ \\
\hline Primigravidas & $1.47(1.23-1.71)^{* * *}$ & $2.91(2.11-4.00)^{* * *}$ & $1.57(1.34-1.83)^{* * *}$ & $3.88(2.83-5.33)^{* * *}$ & $1.48(1.34-1.64)^{* * *}$ & $5.57(4.46-6.97)^{* * *}$ \\
\hline \multicolumn{7}{|l|}{ FGM ${ }^{\mathrm{a}}$ (Ref: no) } \\
\hline Yes & $0.57(0.46-0.72)^{* * *}$ & $0.69(0.51-0.94)^{*}$ & $0.57(0.45-0.71)^{* * *}$ & $0.61(0.44-0.84)^{* *}$ & $0.71(0.61-0.82)^{* * *}$ & $0.93(0.76-1.13)$ \\
\hline \multicolumn{7}{|l|}{ BMI (Ref: normal 18.5-24.9) } \\
\hline High risk $(<18.5, \geq 25)$ & $1.46(1.28-1.67)^{* * *}$ & $1.27(1.02-1.59)^{*}$ & $1.26(1.11-1.42)^{* * *}$ & $1.38(1.13-1.77)^{* *}$ & $1.25(1.13-1.38)^{* * *}$ & $1.44(1.24-1.69)^{* * *}$ \\
\hline \multicolumn{7}{|l|}{ Spatial } \\
\hline \multicolumn{7}{|l|}{ Residence (Ref: urban) } \\
\hline Rural & $0.68(0.61-0.76)^{* * *}$ & $0.78(0.09-6.56)$ & $0.73(0.66-0.81)^{* * *}$ & $0.66(0.12-3.52)$ & $0.77(0.71-0.84)^{* * *}$ & $0.73(0.19-2.68)$ \\
\hline \multicolumn{7}{|l|}{ Region (Ref: urban governorates) } \\
\hline Urban-Lower Egypt & $2.07(1.20-3.56)^{* *}$ & $0.94(0.73-1.21)$ & $1.93(1.19-3.15)^{* *}$ & $1.08(0.81-1.43)$ & $1.93(1.26-2.96)^{* *}$ & $1.16(0.91-1.47)$ \\
\hline Rural-Lower Egypt & $2.21(1.27-3.83)^{* *}$ & $0.99(0.12-8.35)$ & $2.29(1.39-3.75)^{* *}$ & $1.40(0.26-7.61)$ & $2.62(1.70-4.04)^{* * *}$ & $1.08(0.29-4.00)$ \\
\hline Urban-Upper Egypt & $1.45(0.85-2.49)$ & $0.50(0.38-0.67)$ & $1.49(0.92-2.41)$ & $0.90(0.68-1.17)$ & $1.81(1.19-2.74)^{* *}$ & $0.62(0.49-0.77)^{* * *}$ \\
\hline Rural-Upper Egypt & $1.17(0.67-2.03)$ & $0.90(0.10-7.66)$ & $1.68(1.02-2.76)^{*}$ & $1.27(0.23-6.92)$ & $1.28(0.84-1.97)$ & $0.74(0.20-2.75)$ \\
\hline Frontier governorates & $0.87(0.51-1.51)$ & $0.47(0.18-1.22)$ & $1.26(0.77-2.05)$ & $0.56(0.25-1.25)$ & $0.95(0.62-1.44)$ & $0.69(0.33-2.74)$ \\
\hline
\end{tabular}

Newborn characteristics

Birth weight (Ref: high risk $<2,500, \geq 4,000)$

$\begin{array}{lllllll}\text { Normal 2,500-3,999 } \quad 0.75(0.64-0.90)^{* *} & 0.76(0.63-0.91)^{* *} & 0.67(0.56-0.81)^{* * *} & 0.65(0.54-0.79)^{* * *} & 0.92(0.81-1.04) & 0.86(0.75-0.98)^{*}\end{array}$

Birth multiplicity (Ref: multiple)

Singleton $\quad 0.51(0.38-0.68)^{* * * *} \quad 0.44(0.30-0.65)^{* *} \quad 0.67(0.48-0.92)^{* *} \quad 0.50(0.32-0.781)^{* *} \quad 0.52(0.39-0.70)^{* * *} \quad 0.46(0.32-0.66)^{* * *}$

Previous birth c-section ${ }^{\mathrm{b}}$ (Ref: no)

$\begin{array}{lllllll}\text { Yes } & 66.3(44.3-99.2)^{* * *} & 113(58.4-218)^{* * * *} & 61.4(39.6-95.1)^{* * *} & 114.4(55.3-236)^{* * *} & 69.4(47.9-100)^{* * *} & 123.3(71.9-211)^{* * *} \\ \text { Primigravidas } & 1.87(1.64-2.10)^{* * *} & 2.76(2.23-3.41)^{* * *} & 1.90(47.9-100)^{* * *} & 2.83(2.30-3.49)^{* * *} & 2.64(2.38-2.91)^{* * *} & 3.82(3.27-4.46)^{* * *}\end{array}$

Institutional

ANC (Ref: no visits)

$\begin{array}{lllllll}1-3 \text { visits } & 1.56(1.24-2.00)^{* * * *} & 1.58(1.01-2.47)^{* *} & 1.20(0.93-1.56) & 1.18(0.76-1.85) & 1.28(1.04-1.60)^{*} & 0.87(0.61-1.24) \\ \geq 4 \text { visits } & 2.48(2.11-2.90)^{* * * *} & 1.64(1.21-2.23)^{* *} & 1.77(1.52-2.06)^{* * *} & 1.51(1.17-1.94)^{* *} & 2.45(2.10-2.90)^{* * *} & 1.31(1.01-1.70)^{*}\end{array}$

Place of delivery (Ref: public)

Private sector $\quad 1.26(1.13-1.40)^{* * * *} \quad 1.36(1.14-1.62)^{* *} \quad 1.39(1.26-1.55)^{* * * *} \quad 1.40(1.17-1.66)^{* * *} \quad 2.16(1.98-2.36)^{* * * *} \quad 1.96(1.72-2.23)^{* * *}$

Number of cases included in multivariate models was only 12,894 (9236 children were not weighted and 64 mothers did not report on the ANC visits)

OR: odds ratio, aOR: adjusted odds ratio (for all covariates under analysis), Cl: confidence interval, ANC: antenatal care

${ }^{a}$ female genital mutilation, ${ }^{b}$ previous birth occurred within the past five years

${ }^{*} P<0.05,{ }^{* *} P=0.001,{ }^{* * *} P<0.001$ 
was higher than that in EDHS-2005 (aOR, 2.91, 95\% CI: 2.11-4.00). Normal birth weight babies had higher odds undergoing c-section delivery in EDHS-2014 (aOR, 0.86, 95\% CI: 0.75-0.98) compared to EDHS-2005 (aOR, 0.76, 95\% CI: 0.63-0.91).

Furthermore, place of delivery-stratified multivariable logistic regression demonstrated that in private sector, mothers aged $\leq 18$ years had less likelihood to undergo c-sections in EDHS-2005 (aOR, 0.28, 95\% CI: 0.15-0.53) compared to EDHS-2014 (aOR, 0.40, 95\% CI: 0.260.63). However, in EDHS-2014 same age group of mothers was $84 \%$ less likely to undergo c-sections in the public sector. In public sector, SES was not associated with c-section delivery in all survey rounds, but in private sector, the significant association of SES with csection observed in EDHS-2005 disappeared in EDHS2014. The likelihood of primigravida mothers to undergo c-section in public sector declined from 2.21-time (95\% CI: $1.37-3.58$ ) to 1.72 -time (95\% CI: $1.18-2.50$ ) whereas it increased in private sector from 1.62-times (95\% CI: $1.12-2.34$ ) to 3.76-times (95\% CI: 2.94-4.80), in EDHS2005 to EDHS-2014, respectively. Normal birth weight babies were significantly at lower risk to undergo csection in EDHS-2005, but in EDHS-2014 they had an equal likelihood with high-risk birth weight babies in both public and private sectors (Table 5).

\section{Discussion}

The study estimated trend of c-sections among Egyptian mothers sampled in 2005, 2008, and 2014. There was a substantial rise in trend of institutional-based c-sections by more than three-fold, over the study period. The private sector appears to be the driver of the rising csections in Egypt, a substantial increase was also observed in use of this surgical procedure in public sector. The more than 4-folds increase in c-sections in the private sector was driven by substantial increases in csections among mothers who are potentially at low risk for c-section delivery.

In Egypt, the nearly 60\% population-based proportion of c-sections performed in 2014 greatly exceeds the threshold of $10-15 \%$ recommended by WHO [1]. A populationbased proportion of c-sections $>10 \%$ did not lead to health improvements for mother or newborn [33]. Although the observed over time increase in c-section rate in Egypt is in line with what has been noted in many national and international studies [12, 14, 18, 34], this over time increase places Egypt as a country with the highest c-sections performed worldwide, after Brazil (45.9\%) [8]. The institutional-based proportion (67.3\%) of c-sections recorded in Egypt in 2014 is 2.2-time and 2.7-time higher than that recently recorded in Jordan (30.3\%) [14] and in Saudi Arabia (25\%) [35], respectively. The decline in home-based deliveries by over $60 \%$ merely reflects an improvement in provided health care services in Egypt. Over the past decade, per capita total expenditure on health increased from US\$75.8 in 2000 to US\$123.2 in 2010 [36]. However, improving administered health care services should not justify the massive increase in csections. This exponential rise in c-sections indicates an overuse for this surgical procedure that might be due to many c-sections may increasingly be performed without any medical indication.

This rise in c-sections would pose further economic burden in a resource limited-setting such as Egypt, which is already burdened with different economic difficulties where 26.3\% of Egyptians live below the poverty line [37]. In 2008, the WHO estimated that 253,890 unnecessary c-sections had been performed with a total cost of US $\$ 41,085,585$ per year [38]. Referring to the obtained results, the discernible increase in c-sections in Egypt in 2014, this study assumes that the unnecessary c-sections and its associated spending at least would double the ones estimated in 2008 [38]. Furthermore, the increased c-sections would pose further unfavorable health outcomes as a result of adverse outcomes associated with c-sections $[5,6]$ in a country already burdened with a relatively high MMR and NMR in addition to other infectious diseases mainly hepatitis $C$ virus that infect nearly $15 \%$ of the 15 to 59 years old Egyptian people [39]. This disease alone consumes about 20\% of the Ministry of Health and Population total annual budget to treat infected individuals [40]. Rigorous institutional-based study is needed to assess the impact of this high proportion of csections and identify the exact medical and non-medical needs for c-section deliveries for future planning and effective policy interventions.

In the three surveys, childbearing at $\leq 18$ years or $\geq 35$ years, living in high SES, maternal overweight/obesity, pregnancy with high-risk birth weight or multiple babies, delivery in a private sector were found as significant factors associated with c-section delivery in Egypt except for SES in EDHS-2014. Older mothers are more likely to experience different complications during pregnancy and delivery [41-45], even in the absence of complications, and they are more inclined to have c-section, especially primigravida mothers [44]. Younger mothers are more likely to have small pelvis to deliver a fetus which necessitate a c-section [46]. Women with high SES are more likely to be educated and to have higher income who tend to delay giving birth until older age, therefore, increasing their likelihood of a csection delivery [42]. However, the disappeared association of SES with c-sections observed in EDHS-2014 merely explains the penetration of health care services to the socially and economically disadvantaged mothers, nevertheless this should not justify the increased c-sections among this socially deprived group. Maternal overweight/obesity increases the risk of different c-section inducing factors such as preeclampsia and gestational diabetes [47]. 
Table 5 Place of delivery-stratified multivariate logistic regression for the association between institutional-based c-section delivery as dependent variable and measured characteristics, by the survey round

\begin{tabular}{|c|c|c|c|c|c|}
\hline \multicolumn{2}{|c|}{ EDHS-2005 } & \multicolumn{2}{|c|}{ EDHS-2008 } & \multicolumn{2}{|c|}{ EDHS-2014 } \\
\hline Public & Private & Public & Private & Public & Private \\
\hline \multicolumn{2}{|c|}{ aOR $(95 \%$ Cl) } & \multicolumn{2}{|c|}{$\mathrm{aOR}(95 \% \mathrm{Cl})$} & \multicolumn{2}{|c|}{ aOR $(95 \% \mathrm{Cl})$} \\
\hline
\end{tabular}

\section{Socio-demographic}

Age at last birth (Ref: $\geq 35 \mathrm{yrs}$ )

$\begin{array}{llllllll}30-34 & 0.23(0.09-0.62)^{* *} & 0.59(0.41-0.87)^{* *} & 0.70(0.43-1.15) & 0.93(0.63-1.36) & 0.75(0.51-1.10) & 0.79(0.61-1.07) \\ 25-29 & 0.33(0.19-0.54)^{* * *} & 0.40(0.28-0.56)^{* * *} & 0.89(0.57-1.40) & 0.66(0.46-0.93)^{*} & 0.52(0.36-0.74)^{* * *} & 0.56(0.44-0.72)^{* * *} \\ 19-24 & 0.71(0.44-1.14) & 0.31(0.21-0.45)^{* * *} & 0.37(0.23-0.61)^{* * *} & 0.44(0.30-0.64)^{* * *} & 0.39(0.27-0.57)^{* * *} & 0.43(0.33-0.56)^{* * *} \\ \leq 18 & 1.23(0.77-1.98) & 0.28(0.15-0.53)^{* * *} & 0.27(0.11-0.67)^{* *} & 0.43(0.24-0.79)^{* *} & 0.16(0.07-0.35)^{* * *} & 0.40(0.26-0.63)^{* * *}\end{array}$

Socioeconomic status (Ref: low)

\begin{tabular}{|c|c|c|c|c|c|c|}
\hline Medium & $1.31(0.76-2.27)$ & $1.71(1.04-2.83)^{*}$ & $1.23(0.74-2.06)$ & $0.59(0.39-0.88)^{* *}$ & $0.86(0.60-1.22)$ & $1.07(0.83-1.38)$ \\
\hline High & $1.51(0.90-2.55)$ & $2.04(1.26-3.29)^{* *}$ & $1.25(0.76-2.05)$ & $0.61(0.42-0.88)^{* *}$ & $1.11(0.78-1.59)$ & $0.97(0.76-1.25)$ \\
\hline \multicolumn{7}{|l|}{ ifetime parity (Ref: $\geq 4$ ) } \\
\hline $2-3$ & $1.54(1.05-2.26)^{*}$ & $1.04(0.76-1.41)$ & $1.41(0.95-2.07)$ & $2.20(1.60-3.03)^{* * *}$ & $1.41(1.05-1.89)^{*}$ & $1.82(1.51-2.21)^{* * *}$ \\
\hline Primigravidas & $2.21(1.37-3.58)^{* *}$ & $1.62(1.12-2.34)^{*}$ & $1.50(0.92-2.44)$ & $3.73(2.56-5.42)^{* * *}$ & $1.72(1.18-2.50)^{* *}$ & $3.76(2.94-4.80)^{* * *}$ \\
\hline \multicolumn{7}{|l|}{ GMa (Ref: no) } \\
\hline Yes & $0.77(0.41-1.45)$ & $0.68(0.48-0.97)^{*}$ & $0.51(0.24-1.06)$ & $0.62(0.43-0.89)^{*}$ & $0.81(0.55-1.20)$ & $0.95(0.75-1.20)$ \\
\hline \multicolumn{7}{|c|}{ 3MI (Ref: normal 18.5-24.9) } \\
\hline High risk $(<18.5, \geq 25)$ & $1.14(0.78-1.67)$ & $1.34(1.01-1.77)^{*}$ & $1.24(0.88-1.76)$ & $1.52(1.19-1.92)^{* *}$ & $1.78(1.32-2.40)^{* * *}$ & $1.33(1.11-1.60)^{* *}$ \\
\hline \multicolumn{7}{|l|}{ patial } \\
\hline \multicolumn{7}{|l|}{ Residence (Ref: urban) } \\
\hline Rural & $0.82(0.60-10.9)$ & $0.70(0.01-36.73)$ & $0.95(0.10-8.69)$ & $0.52(0.04-7.05)$ & $0.56(0.08-4.04)$ & $1.0(0.14-6.96)$ \\
\hline \multicolumn{7}{|c|}{ Region (Ref: urban governorates) } \\
\hline Urban-Lower Egypt & $1.14(0.73-1.79)$ & $0.91(0.66-1.24)$ & $0.95(0.57-1.62)$ & $1.13(0.79-1.60)$ & $1.04(0.69-1.56)$ & $1.19(0.88-1.60)$ \\
\hline Rural-Lower Egypt & $1.06(0.08-14.7)$ & $1.07(0.02-56.6)$ & $1.29(0.14-12.19)$ & $1.56(0.11-21.4)$ & $1.55(0.21-11.4)$ & $0.74(0.11-5.14)$ \\
\hline Urban-Upper Egypt & $0.34(0.19-0.58)^{* * *}$ & $0.61(0.43-0.86)^{* *}$ & $0.96(0.59-1.54)$ & $0.89(0.64-1.24)$ & $0.62(0.42-0.91)^{*}$ & $0.60(0.45-0.80)^{* *}$ \\
\hline Rural-Upper Egypt & $0.67(0.05-9.46)$ & $1.18(0.02-63.2)$ & $1.27(0.14-11.9)$ & $1.32(0.09-18.2)$ & $1.04(0.14-7.67)$ & $0.51(0.07-3.54)$ \\
\hline Frontier governorates & $0.81(0.24-2.71)$ & $0.25(0.05-1.23)$ & $0.56(0.18-1.77)$ & $0.62(0.19-1.91)$ & $0.72(0.19-2.75)$ & $0.68(0.27-1.73)$ \\
\hline
\end{tabular}

Newborn characteristics

Birth weight (Ref: high risk $<2,500, \geq 4,000$ )

Normal $(2,500-3,999) \quad 0.63(0.47-0.84)^{* *}$

$0.79(0.62-0.99)^{*} \quad 0.46(0.34-0.62)^{* * *} \quad 0.81(0.64-1.04)$

$0.83(0.66-1.05)$

$0.83(0.65-1.04)$

Birth multiplicity (Ref: multiple)

$\begin{array}{lcccccc}\text { Singleton } & 0.39(0.20-0.75)^{* *} & 0.46(0.28-0.77)^{* *} & 1.0(0.47-0.2 .11) & 0.33(0.18-0.60)^{* * *} & 0.57(0.32-1.03) & 0.41(0.26-0.66)^{* * *} \\ \begin{array}{l}\text { Previous birth C-section } \\ \text { (Ref: no) }\end{array} & 53.6(25.8-120.4)^{* * *} & 263.3(82.2-843)^{* * *} & 66.5(25.1-176)^{* * *} & 236.5(73.1-765)^{* * *} & 95.4(41.7-218)^{* * *} & 145.3(71.0-297)^{* * *} \\ \text { Yes } & 2.61(1.81-3.70)^{* * *} & 2.85(2.19-3.72)^{* * *} & 1.84(1.27-2.70)^{* *} & 3.56(2.76-4.60)^{* * *} & 2.48(1.86-3.32)^{* * *} & 4.52(3.75-5.45)^{* * *} \\ \begin{array}{l}\text { Primigravidas } \\ \text { Institutional }\end{array} & & & & & & \\ \text { ANC (Ref: no visits) } & & & & & & \\ 1-3 & 0.92(0.46-1.84) & 2.33(1.27-4.28)^{* *} & 0.72(0.30-1.74) & 1.33(0.77-2.29) & 1.04(0.58-1.84) & 0.78(0.49-1.24) \\ \geq 4 & 1.59(1.03-2.44)^{*} & 1.71(1.11-2.63)^{*} & 1.89(1.29-2.77)^{* *} & 1.29(0.91-1.81) & 1.44(0.98-2.10) & 1.21(0.84-1.74)\end{array}$

Number of cases included in multivariate models was only 12,894 (9236 children were not weighted and 64 mothers did not report on the ANC visits) OR: odds ratio' aOR: adjusted odds ratio (for all covariates under analysis), Cl: confidence interval, ANC: antenatal care

${ }^{\mathrm{a}}$ female genital mutilation, ${ }^{\mathrm{b}}$ previous birth occurred within the past five years

${ }^{*} P<0.05,{ }^{* *} P=0.001,{ }^{* * *} P<0.001$ 
One of the principal objectives of this investigation was to identify main drivers of the increased c-sections in Egypt. Study findings documented that place of delivery is a major contributor to the exponentially increased c-sections in Egypt. Although the private sector occupies only $16 \%$ of total hospitals beds in Egypt [15], the trend of c-sections increased by more than 4-time over the study period. This finding is still persistent in Egypt and in line with the previous study reported in 2004 and analyzed comparably collected data [18]. Lack of compliance with regulations by private practitioners and inadequate enforcement of the law, public's perception that medical services in private sector due to the availability of necessary medical technology and better inpatient quality care services for this surgical intervention, particularly in presence of near birth complications, as well as obstetricians' predisposition to manage their time, are suggested $[18,23]$ and documented factors associated with the increasing c-sections in the private sector $[31,43,48]$.

The study found that this sizeable rise in proportion of c-sections in the private sector was driven by increasing c-sections among mothers who are theoretically and empirically known as not at risk of csection including mothers who fallen in an age group of 25-29 years, mothers of normal BMI, and mothers reported normal birth weight or singleton babies. This increase contributed substantially to the overall increase in c-sections in Egypt which supports the notion that a sizeable proportion of performed c-sections might be performed unnecessarily. This is in line with what has been recently reported from nationally-representative data in Jordan [14]. According to which, the rising in c-sections from $18.2 \%$ in 2002 to $30.3 \%$ in 2012 , driven primarily by substantial rises among apparently low-risk mothers; mothers with normal birth weight or singleton babies [14].

It is worth to be mentioned that encouragement of vaginal delivery is very important to curb the steady rise in csections in Egypt. This study observed a decline by $12.8 \%$ in repeated institutional-based vaginal deliveries among mothers reported two births in five years preceded the survey. External cephalic version (turning the fetus from a breech or transverse position into a vertex position), vaginal birth after a previous c-section, and one-on-one trained support during labor were effective psychosocial and structural strategies at reducing the likelihood of csections, even among those who may have a medical indication [49]. Moreover, initiatives to raise peoples' and health professionals' awareness about the adverse outcomes associated with c-section and advantages of vaginal delivery are also urgently needed. Educating mothers about risks associated with c-section, midwifery training, and establishment of birthing centers, could also help encourage mothers to deliver vaginally.

The strengths of this study are that the data were from large, randomly selected population-based three datasets collected by accredited and reliable official entities using comparable methodology. The larger sample size and high response rates provided in the study with good statistical power and objective outcome measures. However, in population-based survey, the probability of recall bias is low since mothers who had a c-section delivery would not easily forget the mode of delivery given its surgical nature, particularly for the last birth [19]. Estimates associated with c-section were adjusted for any potential confounding effect of the measured characteristics. Stratification according to the place of delivery provided more insights about the substantial contribution of the private sector to the rising c-sections in Egypt with revealing contributing factors for the increased c-sections in this sector.

Findings from this study should be interpreted in light of the following limitations. The cross-sectional design of the EDHS limits the causality pathway with regard to the factors found associated with increased c-sections. Given the nature of the household-based survey where medical records are usually unavailable, collected data did not include information about whether the c-sections were performed under medical indications such as fetal mal-presentation or based solely on maternal demand, except for the birth weight and birth multiplicity that served as the only obstetric indicators could potentially at medically necessary c-section. Despite of these limitations, this study provided evidence-based estimates on trend of c-section deliveries in Egypt and associated factors to fine-tune strategies necessary to halt the rising csections in Egypt.

\section{Conclusions}

Obtained results demonstrated that the proportion of csections in Egypt has been increasing steadily in recent years and has reached an alarming level. The proportion of c-sections documented in the last EDHS conducted in 2014 quadrupled the maximum threshold recommended by the WHO. The increase in number of birth deliveries occurred in the private sector appears to be associated with a shift towards delivery in private facilities. This increase in the private sector, particularly among mothers who were potentially at low risk of c-sections requires an urgent need to adopt critical policies and strategies that able to halt the steady rise in c-sections in Egypt and improve reproductive health and mothers and babies health outcomes. In the meantime, an in-depth institutionalbased study collecting data on the exact indications associated with c-sections in Egypt is also necessary. 


\section{Additional file}

Additional file 1: STROBE checklist. STROBE statement. A checklist statement summarizing reporting of observational studies. (DOC $130 \mathrm{~kb}$ )

\section{Abbreviations}

ANC: Antenatal care; aOR: Adjusted odds ratio; Cl: Confidence interval; Csection: Caesarean section; DHS: Demographic and health surveys; EDHS: Egypt Demographic and Health Surveys; MMR: Maternal mortality ratio; NMR: Neonatal mortality rate; OR: Odds ratio; SES: Socioeconomic status; STROBE: Strengthening the Reporting of Observational Studies in Epidemiology; WHO: World Health Organization

\section{Acknowledgements}

Author is very grateful to the EDHS for collecting data. In addition, he would like to acknowledge all individuals and institutions in Egypt involved in the implementation of the 2005, 2008, and 2014 EDHS. The statements made herein are solely the responsibility of the author.

\section{Funding}

This work received no specific funding. Author is grateful to the College of Medicine and Health Sciences at the United Arab Emirates University, for covering the publication fees. The statements made herein are solely the responsibility of the author.

\section{Availability of data and materials}

The EDHS data are accessible from the Measure DHS website [20].

\section{Author's contributions}

RA, conceptualized and designed the study, analyzed the data, and drafted and critically wrote the article.

\section{Ethics approval and consent to participate}

This study is based solely on a retrospective analysis of secondary existing anonymous survey data published by the DHS program. EDHS is approved by the government of Egypt. Data collection team obtained voluntary consent from sampled women to participate in the survey, study protocol and consent procedure are described elsewhere [16]. For this analysis, there was no need for permission from the institutional review board.

\section{Consent for publication}

Not applicable.

\section{Competing interests}

The author declares that he has no competing interests.

\section{Publisher's Note}

Springer Nature remains neutral with regard to jurisdictional claims in published maps and institutional affiliations.

Received: 18 July 2016 Accepted: 23 November 2017 Published online: 13 December 2017

\section{References}

1. World Health Organization. Statement on Caesarean Section Rates. (http:// apps.who.int/iris/bitstream/10665/161442/1/WHO_RHR_15.02_eng.pdf) Accessed 12 Apr 2016.

2. World Health Organization. Cesarean section without medical indication increases risk of short-term adverse outcomes for mothers. Geneva: WHO. Available from: http://whqlibdoc.who.int/hq/2010/WHO_RHR_HRP_10.20_ eng.pdf. Accessed 21 Mar 2015.

3. Souza JP, Gulmezoglu A, Lumbiganon P, Laopaiboon M, Carroli G, Fawole B, et al. Caesarean section without medical indications is associated with an increased risk of adverse short-term maternal outcomes: the 2004-2008 WHO global survey on maternal and Perinatal health. BMC Med. 2010;8:71 doi: https://doi.org/10.1186/1741-7015-8-71. PubMed PMID: 21067593; PubMed Central PMCID: PMCPMC2993644

4. Villar J, Carroli G, Zavaleta N, Donner A, Wojdyla D, Faundes A, et al. Maternal and neonatal individual risks and benefits associated with caesarean delivery: multicentre prospective study. BMJ. 2007;335(7628):1025 doi: https://doi.org/10.1136/bmj.39363.706956.55. PubMed PMID: 17977819: PubMed Central PMCID: PMCPMC2078636.

5. Kennare R, Tucker G, Heard A, Chan A. Risks of adverse outcomes in the next birth after a first cesarean delivery. Obstet Gynecol 2007;109(2 Pt 1): 270-276. doi: https://doi.org/10.1097/01.AOG.0000250469.23047.73. PubMed PMID: 17267823

6. Daltveit AK, Tollanes MC, Pihlstrom H, Irgens LM. Cesarean delivery and subsequent pregnancies. Obstet Gynecol 2008;111(6):1327-1334. doi: https:// doi.org/10.1097/AOG.0b013e3181744110. PubMed PMID: 18515516

7. Allen VM, O'Connell CM, Farrell SA, Baskett TF. Economic implications of method of delivery. Am J Obstet Gynecol 2005;193(1):192-197. doi: https:// doi.org/10.1016/j.ajog.2004.10.635. PubMed PMID: 16021078.

8. Gibbons L, Belizan JM, Lauer JA, Betran AP, Merialdi M, Althabe F. Inequities in the use of cesarean section deliveries in the world. Am J Obstet Gynecol. 2012;206(4):331 e1-331 19. doi: https://doi.org/10.1016/j.ajog.2012.02.026. PubMed PMID: 22464076.

9. Scioscia M, Vimercati A, Cito L, Chironna E, Scattarella D, Selvaggi LE. Social determinants of the increasing caesarean section rate in Italy. Minerva Ginecol 2008:60(2):115-120. PubMed PMID: 18487961

10. McCallum C. Explaining caesarean section in Salvador da Bahia, Brazil Sociol Health IIIn 2005;27(2):215-242. doi: https://doi.org/10.1111/j.1467-9566.2005. 00440.x. PubMed PMID: 15787776.

11. Nuttall C. Caesarean section controversy. The caesarean culture of Brazil. BMJ. 2000;320(7241):1074. PubMed PMID: 10836808

12. Ba'aqeel HS. Cesarean delivery rates in Saudi Arabia: a ten-year review. Ann Saudi Med. 2009;29(3):179-183. PubMed PMID: 19448379; PubMed Central PMCID: PMCPMC2813649.

13. Giani U, Bruzzese D, Pugliese A, Saporito M, Triassi M. [Risk factors analysis for elective caesarean section in Campania region (Italy)]. Epidemiol Prev 2011;35(2):101-110. PubMed PMID: 21628753.

14. Al Rifai R. Rising cesarean deliveries among apparently low-risk mothers at university teaching hospitals in Jordan: analysis of population survey data, 2002-2012. Glob Health Sci Pract. 2014;2(2):195-209. doi: https://doi.org/10. 9745/GHSP-D-14-00027. PubMed PMID: 25276577; PubMed Central PMCID: PMCPMC4168617.

15. Ministry of Health and Population (MOHP) [Egypt] E-ZA, and ORC Macro. Egypt service provision assessment survey 2002. Calverton, Maryland: Ministry of Health and Population, El-Zanaty Associates, and ORC Macro. Available from: https://dhsprogram.com/pubs/pdf/SPA5/SPA5.pdf. Accessed 20 Apr 2016.

16. Ministry of Health and Population [Egypt] E-ZaAE, and ICF International. Egypt demographic and health survey 2014. Cairo, Egypt and Rockville, Maryland, USA: Ministry of Health and Population and ICF International. 2015.

17. UNFPA. UNFPA-Egypt. Available from: http://egypt.unfpa.org/ Accessed 2 Apr 2016.

18. Khawaja M, Jurdi R, Kabakian-Khasholian T. Rising trends in cesarean section rates in Egypt. Birth. 2004;31(1):12-16. PubMed PMID: 15015988; PubMed Central PMCID: PMCPMC1457115.

19. Stanton CK, Dubourg D, De Brouwere V, Pujades M, Ronsmans C. Reliability of data on caesarean sections in developing countries. Bull World Health Organ. 2005:83(6):449-455. doi: /S0042-96862005000600013. PubMed PMID: 15976896; PubMed Central PMCID: PMCPMC2626266

20. Measure. Demographic and Health Surveys. Measures DHS. 2010

21. STROBE Statement-Checklist of items that should be included in reports of cross-sectional studies. Availbale from: http://www.strobe-statement.org/ fileadmin/Strobe/uploads/checklists/STROBE_checklist_v4_cross-sectional. pdf. Accessed 20 Nov 2015.

22. Rutstein S, Rojas G. Guide to DHS statistics. Demographic and Health Surveys ORC Macro, Calverton, Maryland. 2006. Available from: http:// citeseerx.ist.psu.edu/viewdoc/download?doi=10.1.1.431.8235\&rep= rep1\&type=pdf. Accessed 22 Mar 2016

23. Khawaja M, Al-Nsour M. Trends in the prevalence and determinants of caesarean section delivery in Jordan: evidence from three demographic and health surveys, 1990-2002. World Health Popul 2007:9(4):17-28. PubMed PMID: 18567949

24. Rahman M, Nakamura K, Kizuki M. Socioeconomic differences in the prevalence, awareness, and control of diabetes in Bangladesh. J Diabetes Complicat 2015;29(6):788-793. doi: https://doi.org/10.1016/j.jdiacomp.2015. 04.011. PubMed PMID: 25981122 
25. Organization WH. Physical status: the use and interpretation of anthropometry. Geneva, Switzerland: WHO (http://whqlibdoc.who.int/trs/ WHO_TRS_854.pdf). Accessed 30 March, 2015.

26. Hoellen F, Hornemann A, Haertel C, Reh A, Rody A, Schneider S, et al. Does maternal underweight prior to conception influence pregnancy risks and outcome? In Vivo 2014;28(6):1165-1170. PubMed PMID: 25398817.

27. Leung TY, Leung TN, Sahota DS, Chan OK, Chan LW, Fung TY, et al. Trends in maternal obesity and associated risks of adverse pregnancy outcomes in a population of Chinese women. BJOG 2008;115(12):1529-1537. doi: https:// doi.org/10.1111/j.1471-0528.2008.01931.x. PubMed PMID: 19035989.

28. Liu Y, Dai W, Dai X, Li Z. Prepregnancy body mass index and gestational weight gain with the outcome of pregnancy: a 13-year study of 292,568 cases in China. Arch Gynecol Obstet 2012;286(4):905-911. doi: https://doi. org/10.1007/s00404-012-2403-6. PubMed PMID: 22695822.

29. Zhang Y, Wang ZL, Liu B, Cai J. Pregnancy outcome of overweight and obese Chinese women with gestational diabetes. J Obstet Gynaecol 2014; 34(8):662-665. doi: https://doi.org/10.3109/01443615.2014.920787. PubMed PMID: 24911378

30. Ding XX, Xu SJ, Hao JH, Huang K, Su PY, Tao FB. Maternal pre-pregnancy BMI and adverse pregnancy outcomes among Chinese women: results from the C-ABCS. J Obstet Gynaecol 2016;36(3):328-332. doi: https://doi.org/10. 3109/01443615.2015.1050652. PubMed PMID: 26492517.

31. Gomes UA, Silva AA, Bettiol H, Barbieri MA. Risk factors for the increasing caesarean section rate in Southeast Brazil: a comparison of two birth cohorts, 1978-1979 and 1994. Int J Epidemiol 1999;28(4):687-694. PubMed PMID: 10480697.

32. Corporation. I. IBM SPSS statistics for windows, version 22.0. Armonk, New York: IBM Corporation; 2013. 2013.

33. Souza JP, Betran AP, Dumont A, de Mucio B, Gibbs Pickens CM, DeneuxTharaux C, et al. A global reference for caesarean section rates (C-model): a multicountry cross-sectional study. BJOG 2016;123(3):427-436. doi: https:// doi.org/10.1111/1471-0528.13509. PubMed PMID: 26259689.

34. Subedi S. Rising rate of cesarean section-a year review. Journal of Nobel Medical College. 2012;1(2):72-6

35. Wahabi H, Fayed A, Esmaeil S, Alzeidan R, Elawad M, Tabassum R, et al. Riyadh mother and baby multicenter cohort study: the cohort profile. PLoS One. 2016;11(3):e0150297. doi: https://doi.org/10.1371/journal.pone.0150297. PubMed PMID: 26937965; PubMed Central PMCID: PMCPMC4777404.

36. World Health Organization. Country statistics and global health estimates by WHO and UN partners. Egypt: WHO statistical profile. Last updated: January 2015. Available from: http://www.who.int/gho/countries/egy.pdf?ua=1. Accessed 10 May 2016.

37. UNDP. UNDP human development report 2015, Egypt central Agency for Public Mobilization and Statistics - CAPMAS. 2015.

38. Luz Gibbons JMB, Jeremy A Lauer, Ana P Betrán, Mario Merialdi and Fernando Althabe. The Global Numbers and Costs of Additionally Needed and Unnecessary Caesarean Sections Performed per Year: Overuse as a Barrier to Universal Coverage. World Health Report (2010) Background Paper, 30. Available from: http://www.who.int/healthsystems/topics/ financing/healthreport/30C-sectioncosts.pdf. Accessed 10 Jan 2016.

39. El-Zanaty FaAW. Egypt demographic and health survey 2008. Cairo, Egypt: Ministry of Health, El-Zanaty and Associates, and Macro International. 2009

40. World Health Organization. World Hepatitis Day - July 28, 2012. MMWR: Morbidity \& Mortality Weekly Report 61: 1/3p. 2012.

41. Bell JCD, Graham WJ, Penney GC, Ryan M, Hall MH. Do obstetric complications explain high caesarean section rates among women over 30 ? A retrospective analysis. Br Med J. 2001;322:894-5.

42. Mishra US, Ramanathan M. Delivery-related complications and determinants of caesarean section rates in India. Health Policy Plan 2002;17(1):90-98. PubMed PMID: 11861590

43. Padmadas SS, Kumar S, Nair SB, Kumari A. Caesarean section delivery in Kerala, India: evidence from a National Family Health Survey. Soc Sci Med 2000;51(4):511-521. PubMed PMID: 10868667.

44. Peipert JF, Bracken MB. Maternal age: an independent risk factor for cesarean delivery. Obstet Gynecol 1993;81(2):200-205. PubMed PMID: 8423950.

45. Webster LA, Daling JR, MCFarlane C, Ashley D, Warren CW. Prevalence and determinants of caesarean section in Jamaica. J Biosoc Sci 1992;24(4):515525. PubMed PMID: 1429779

46. Nour NM. Health consequences of child marriage in Africa. Emerg Infect Dis. 2006;12(11):1644-1649. doi: https://doi.org/10.3201/eid1211.060510. PubMed PMID: 17283612; PubMed Central PMCID: PMCPMC3372345.
47. Leddy MA, Power ML, Schulkin J. The impact of maternal obesity on maternal and fetal health. Rev Obstet Gynecol. 2008;1(4):170-178. PubMed PMID: 19173021; PubMed Central PMCID: PMCPMC2621047.

48. Platt MJ, Marshall A, Pharoah PO. The effects of assisted reproduction on the trends and zygosity of multiple births in England and Wales 1974-99. Twin Res 2001:4(6):417-421. PubMed PMID: 11780931.

49. Walker R, Turnbull D, Wilkinson C. Strategies to address global cesarean section rates: a review of the evidence. Birth 2002;29(1):28-39. PubMed PMID: 11843787.

\section{Submit your next manuscript to BioMed Central and we will help you at every step:}

- We accept pre-submission inquiries

- Our selector tool helps you to find the most relevant journal

- We provide round the clock customer support

- Convenient online submission

- Thorough peer review

- Inclusion in PubMed and all major indexing services

- Maximum visibility for your research

Submit your manuscript at www.biomedcentral.com/submit
) Biomed Central 